\title{
On the representation of change according to different ontologies of learning
}

\author{
Miguel-Ángel Sicilia
}

Computer Science Department, University of Alcalá,

Ctra. Barcelona, km. 33.6 - 28871 Alcalá de Henares (Madrid), SPAIN

E-mail: msicilia@uah.es

\section{Miltiadis D. Lytras}

Department of Management Science and Technology, ELTRUN - The Research Center, Athens University of Economics and Business, 47A Evelpidon Str., 11362 Athens, GREECE

E-mail: mdl@eltrun.gr

\begin{abstract}
Recent work has reintroduced ontology as a research topic in learning theories, as a mean to make explicit the differences and links between existing approaches to the design of learning programs. In the context of technology-supported information systems, ontologies can be represented in machine-understandable form to serve as a basis for automation and assessment. The notion of change is in some form part of any ontology of learning, but the interpretations attributed to the term differ between them both in scope and characterization. Since change is central to the evolving behaviour of learning organizations, it is worth the effort of specifying it, especially for the sake of objective measurement and automation. This paper describes ontological structures for generic constructivist and socio-cultural learning frameworks, stating the differences in their overall concepts of change, and their implications for practice and assessment. The ontological definitions provided are intended to motivate further work in more specific approaches for learning technology-supported experiences.
\end{abstract}

Keywords: Learning, change, ontology, ontology of learning, learning system, constructivist learning, socio-cultural learning.

\section{Introduction}

The work of Packer and Goicoechea (2000) has reintroduced ontology as a meaningful topic in research on learning and development. In their comparative study on the ontological differences between the socio-cultural and constructivist perspectives of learning, they go beyond epistemological matters to analyze the ontological assumptions that underlie both theories. Such kind of clarifications result in conceptualizations that determine the possible and desirable learning approaches and that serve as guidance for research programs and interpretive assessments. Ontology as a discipline that produces 


\section{M.A. Sicilia \& M. Lytras}

conceptual models for concrete aspects of reality makes the foundational principles of designs explicit, and thus, comparable and measurable.

In the context of information systems, the "ontology of learning" - explicitly or tacitly assumed - actually constraints and drives the behaviour of the system as a whole, and the criteria used for the evaluation of the outcomes of activities also become a reflection of previous ontological commitments. For example, a consideration of social aspects of learning will lead to the use of Web-based tools that put an emphasis in structured collaboration, and the post-activity assessments would take into account participation and relationship building as measures of value. In the case of information systems in which automation through software technology is intended to play a significant role, the ontology (or ontologies) of learning assumed should ideally be represented in machine-understandable form. Ontology engineering (Gruber, 1995) provides a method to develop such artefacts, and Semantic Web technology (Berners-Lee, Hendler and Lassila, 2001) aims at providing tools and techniques to develop software that exploits them in the context of a learning system ${ }^{1}$. Formal ontology provides the knowledge representation infrastructure for ontologies of learning to a level of considerable complexity and richness, as supported by description logics (Baader et al,, 2003), and ontology mapping techniques (Noy and Musen, 2003) could eventually used in an attempt to reconcile different ontological views. In what follows, we use the term ontology to refer to the representations in machine-readable form, rather than to use it to argument philosophical positions.

Recent approaches to applying Semantic Web techniques to learning settings (Stutt and Motta, 2004; Allert, 2004) have raised the need for ontological representation of different theories of learning, which in turn can be used to explicitly represent concrete learning designs in connection to ontology terms and relations. Such explicit representations are critical for revealing the underlying assumptions made by tutors or course designers, which would eventually result in extending the post-activity assessments to cover also the appropriateness of the general ontological approach to the given situation. In an ideal setting, in which making explicit learning approaches through ontologies were common practice, researchers would have the opportunity to investigate the overall effectiveness of theoretical design perspectives on a much more precise and sound basis than today. In any case, making explicit the assumptions of the underlying learning theories provides a number of potential benefits, including the following:

- Enables the linking of theoretical assumptions to practical learning designs for informative purposes, since they somewhat trace to the source of the decision-making in the process of crafting learning programs or activities.

- In addition to being an important source for scientific inquiry, such links are by themselves useful resources for education or training of learning designers.

- Explicit assumptions may eventually lead to finding patterns in design situations, useful to inform new designs or even to build decision-aid tools. The necessity for this has been pointed out by Koper (2004), and approaches like the one described by Inaba et al. (2000) are good examples for automation settings that require detecting such patterns.

- Detailed comparisons between the effectiveness and adequacy of learning designs are facilitated by the more precise descriptions of the elements and hypothesis considered. 


\section{Representation of change - ontologies of learning}

The representation of "units of learning" was addressed in the so-called Educational Modelling Language (EML, 2000), which has been replaced by the IMS Learning Design (LD, 2003) specification that was released in February, 2003. LD uses a semantic conceptual model of the teaching-learning process, in which the notion of activity is central. Nonetheless, the activity-centred ontology implicit in LD (Allert, 2004) is deliberately general-purpose, so that it tends to hide the differences in perspective coming from diverging theoretical assumptions on learning.

In any case, the concept of change is central to any ontology of learning, since every model of human learning includes the notion of change in the learners or in the social world, be it a change in knowledge, attitudes, structure or identity. From the standpoint of organizations, learning is both a form of detecting and correcting errors, and also of questioning the organizational processes and inquiring for more adequate ones (Appelbaum, St-Pierre and Glavas, 1998). In consequence, the characterization and measurement of change is critical from the viewpoint of analyzing the evolution of organizations and the individuals inside them. For example, if an approach to intellectual capital statement like the 3R model (Ordóñez de Pablos, P., 2004) is adopted, the human capital part of the intellectual capital flow report should reflect change as the final result of learning activities. Due to its importance for practice and management, in this paper we focus on the parts of the ontologies of learning that affect to change as an objective of learning in its various forms. As will be discussed, different perspectives on learning lead to different accounts of change, so that even the accountability of learning outcomes is finally influenced by the ontology of learning assumed.

Existing ontological representations for learning processes describe in detail educational resources and activities. In addition, some existing ontologies go beyond the activity-based paradigm and describe aspects of a theory, for example, Barros et al. (2003) represent Activity Theory elements, including the steps of conversational structures. Nonetheless, the notion of change is not explicit in existing research that uses formal ontologies to describe learning activities, even though the measures that would eventually be used to characterize the topic are present. Without a characterization of change, it is difficult to communicate the actual design decisions that were integrated in the learning design, since decisions should be founded in some concept of change. In addition, neglecting the explicit definition of change makes difficult the comparison of learning designs and the eventual mining and meta-analysis of interesting patterns as described by Koper (2004). In our view, this is a major flaw of current learning modelling languages if the potential benefits described above are required.

In this paper, the overall elements modelling change inside different ontological views of learning are described. The ontology described here is structured in terms of concepts, relations and axioms (typed in Courier font to differentiate them), following approaches to ontology used elsewhere (Holsapple and Joshi, 2004). For clarity, we have avoided to describe here the formal logics-based version of the ontologies, which have been developed using the OWL language with the ontology editor Protégé2. The ontology fragments described here may be expanded, improved or even replaced by further studies, but they serve as a foundation for a disciplined approach to document the assumptions made in concrete learning designs. It is our intention to motivate further work and discussion on formal, shared ontologies of learning. 


\section{M.A. Sicilia \& M. Lytras}

The rest of this paper is structured as follows. Section 2 sketches the essential elements of generic ontologies of learning from the constructivist and socio-cultural perspectives. Then, Section 3 provides the details of different concepts of change that can be attributed to each theoretical perspective, with an emphasis in providing objective measures for assessment of the outcomes of learning processes. Finally, Section 4 concludes providing a discussion of some of the main implications of the adoption of explicit ontology-based approaches to learning design, and sketches some potential directions for further research.

\section{Representation of Socio-Cultural and Constructivist Ontologies of Learning}

The ontologies sketched in this section are schematic, in the sense that they only intend to provide a basic account of the process of learning and their most relevant surrounding aspects. In practical situations, such kind of ontology would require integration with other ontologies describing the domains that are the subject of the learning experience. For example, mathematics in schooling has some concrete ontological commitments that should be accounted for (Packer and Hwang, 1999). We will first provide basic ontological elements common to any learning design, and then the main, generic elements of constructivist and socio-cultural views on learning will be described. Ontology terms and relations are typed in Courier font to facilitate their identification, and boxes summarize the main concepts. It should be noted that the emphasis on representation of ontologies as a tool for developing information systems makes irrelevant discussing about aspects that transcend pragmatic issues, e.g. the world-learner realism.

\subsection{A Basic Ontology of Learning}

From a naive, information-transmission perspective of learning, Information can be considered as a collection of discrete elements that are stored in a Learner's mind, without considering any specific structure beyond the relationships of the contents themselves. In this view, Learning becomes the act of storage of the content encoded in a Message communicated through some Channel. This basic setting is consistent with Shannon's theory of communication (Weaver and Shannon, 1949), and despite its obvious limitations, it is still assumed in many Web-based learning settings in which simply contents are put on-line for students to read (decode) it. In addition, this ontology serves here the purpose of comparison point with richer ones.

B01: In an information-communication perspective of Learning, Information is communicated through a Channel in the form of Messages. The roles of Source and Target are often equated to the common roles of Tutor and Learner.

The Tutor is often the source of the message, and the channel determines the type of information communicated, e.g. speech, images or video. The essential action in this model is unidirectional, asymmetric communication, which can be modelled through events CommunicationEvent(Message, Tutor, Learner, Channel). Within this ontology, the concept of Learningobject (Polsani, 2003) can be 


\section{Representation of change - ontologies of learning}

modelled as a specific kind of complex message oriented to be used in learning events that uses the Web for its delivery and that is properly described by metadata.

The effect of unidirectional communication may eventually produce an event of learning, which in this view can be considered as an StorageEvent(Information, Learner, CommunicateEvent). It is assumed in this model that communication events are the only activities that actually take place, and the prerequisites to understand the message can be considered as relationships between messages.

BO2: In an information-communication perspective of Learning, the outcomes of the learning system are measured by the number CommunicateEvent instances issued that result in StorageEvent instances, i.e. successful message encoding and decoding (free of Noise).

A consequence of the basic ontology just described is that inefficiencies should be attributed to Noise in the channel, i.e. difficulties of any kind in the communication. This model provides a restrictive view on organizational learning, limited to individual activities that are isolated from context and culture.

\subsection{An activity-centred Ontology of Learning}

The introduction of activity-based elements in the basic ontology described above can be done by examining the LD specification (LD, 2003). In this framework, a learning design is made up of Activities performed by persons assuming a Role. The notion of role is a flexible replacement for the uni-directional communication of the basic ontology, and may represent any kind of involvement in activities. Activities thus generalize simple communication events to interaction episodes possibly involving several artefacts and participants.

AC01: Persons playing a Role become involved in structured learning Activities. An activity may aggregate sub-activities of a diverse kind.

Activities can be further structured in sub-activities to an arbitrary level of complexity (specific kinds of activities can be differentiated, but this is irrelevant to our current discussion). The role-part(Activity, Role) relation represents a specific part of the learning design that can be further structured in increasing levels of complexity named Act, Play and Method. This allows for combining activities with different Conditions on the roles, which act as logical conditions required for roles to engage in specific parts of the learning design. Methods are intended to meet concrete learning objectives and may have some Prerrequisites.

ACO2: Activities played by Roles can be structured through the concepts of Act, Play and Method. Conditions express the requirement imposed on roles and persons to participate in specific parts of the learning design. Methods are designed towards concrete Objectives and may have some Prerrequisites.

Activities can be further described by their Environment, made up of Learningobjects and Services, being the former reusable units of (possibly interactive) content - for a general discussion on the concept, see (Polsani, 2003) -, and 
the latter general facilities that can be accessed through URLs, e.g. a discussion forum or a search tool. The Outcomes of the activity are a record of the results of the process of guided interaction of roles with the environment, and they should be consistent with the objectives of the overall method or of the concrete activity.

ACO3: Activities use an Environment in which Learning Objects and Services are arranged to create some Outcomes.

The just described activity schema is pedagogically neutral, in the sense that it can represent learning designs coming from any other ontology of learning. For example, if we define activities with two asymmetric roles source and target in which the environment contains only an expositive learning object, the resulting simplified activity structure fits the information-processing scheme described above. In fact, as will be mentioned later, this activity framework is able to model the interactions of both constructivist and socio-cultural assumptions. But despite its flexibility, it only reflects the final outcome of the design, in the form of a Method that captures the sequencing of activities to be carried out, not covering the recording of the assumptions that lead to such method and no other. As stated in the introduction to this paper, there are reasons to extend this framework with an explicit statement regarding the decision-making in the design process.

\subsection{Fundamental Elements of the Constructivist Ontology}

Constructivist theories assume the active role of the learner, discarding mere storage of knowledge facts. There are many varieties of constructivism (Raskin, 2002) with some degree of divergence, so that the elements described here should be considered as an oversimplification of the diversity of constructivist approaches, focused on the characterization of constructivism provided by Packer and Goicoechea (2000).

The first assumption of the constructivist ontology is that there exists some form of knowledge structures, which go beyond simple linear information storage and are internal, in contrast to competencies, which are externally observable behaviour. The initial state of such structure influences the outcomes of the result of learning activities, and as such, they should be considered to describe pre-requisites also. An example of the modelling of such structures can be found in the experiments on mapping hypertext to semantic networks (Jonassen and Wang, 1993). This assumption leads to an intensive use of knowledge representations as a fundamental element of the learning design. The concrete type of knowledge representation is actually the realization of the theoretical assumptions of the designer about the structure of mental representations. Ontologies can be used as a general-purpose logics-based substrate for such mapping, and they can be integrated with standards and common practice (Sicilia and García, 2004).

CO1: Each Learner engaged in activities constructs its own Knowledge as part of its internal Knowledge Structure.

A second important assumption is that knowledge is constructed and not transmitted, so that a source-message-target model is not longer valid. Thus, the Environment of the activities play a prominent role, and it should ideally provide a realistic Context for the knowledge to be acquired, as close as possible to reality. This entails that tutors should change their role to become facilitators but not directors of the learning process. 


\section{Representation of change - ontologies of learning}

CO2: The Context of the activities should provide a realistic setting to the Environment of activities, and the activities should provide different approaches to the same content, in order to provide alternate paths to approach knowledge construction. Tutors become Coaches that participate but not constrain the learning process.

The constructivist ontology described so far can be realized through activity centred designs, but constraining the arrangement of activities in several dimensions, including: (a) connecting the activities to the knowledge structures that are intended to reflect or work on, (b) providing alternative views on the reality being discussed (i.e. multiple alternative Methods or variations inside them), and (c) giving the tutor a coaching role. The provision of alternate views or access to surrounding concepts can be realized in the Web with ontology-based tools that incorporate some tactics for casual discovery (García and Sicilia, 2003).

One last but not least important characteristic of the constructivist ontology is that not all knowledge built is of equal importance, i.e. there are levels of knowledge. This sentence raises the problem of which and how many are the levels to be considered, but there is not a shared, uncontroversial answer to this question, so that it should be left to the particular assumptions made by the learning designer.

CO3: Knowledge Structures have different levels that are qualitatively different.

The problem of devising an explicit approach to a constructivist view of learning then entails the definition of a concrete cognitive structure and its dynamic forms of evolution that accommodates the theoretical assumptions used as a point of departure for the learning design. For example, conceptions of adaptiveness like the one described by Christensen and Hooker (2000) can be translated to a logical representation as a explicative framework for developing an operational account of knowledge construction.

\subsection{Fundamental Elements of the Socio-Cultural Ontology}

Theories of learning emphasizing the socio-cultural aspects view cognition as a complex social phenomenon, mediated by participation in social activities, in which the learner is to some extent guided. This generic characterization includes two of the most prominent elements that influence learning designs: Social Context and "secondary" participation. Consequently, relationships (of a diverse kind) built or changed as a result of the interactions in learning activities are not a secondary product but a fundamental outcome of social participation.

SC1: Socio-cultural learning settings consider the Social Context as the fundamental environment in which activity takes place, and learners engage in such activities as participants among other roles. Relationships between participants are both a driver and a result of interaction in learning.

Social contexts must not be confused with the contextualization of activities in reality described in the constructivist ontology, since they are a specific setting in which the Culture of the group determines membership. The concept of Role described in the activity-centred paradigm should then be specialized to cover the specific roles that are 


\section{M.A. Sicilia \& M. Lytras}

integral to each kind of social context. For example, the interactions allowed to a learner in approaches that assume peripheral participation (Lave and Wenger, 1990) should be oriented to progressive integration in full-membership roles from roles of the kind of Apprentice. In addition, the Culture of the social setting should be subject to modelling, since learning is also a matter of acquiring its norms and system of beliefs. For the latter, ontologies or similar representations could also be used.

SC2: The Culture of the group determines learning, so that it is important to describe its Norms and Beliefs, among other constituent elements.

The consideration of culture introduces a complex element, since it should be considered that cognition affects the group and not only the individual.

Activity-oriented frameworks allow for the representation of norms as Conditions on activities that should be met by individuals. In this way, the process of acquiring an Identity inside the social group constitutes the fundamental thread that guides learning.

SC3: The learner changes in its conditions and even roles in the social group, thus constructing an Identity that determines its responsibilities.

In addition, the social nature of learning entails some degree of self-guiding of the group. This may eventually be materialized in the group arranging new objectives for learning - as sub-goals for the overall goal of the learning program - in a collaborative manner. Although this may also be applicable to constructivist designs, the convergence of the aptitudes, knowledge and interests of many participants would make it more likely in socio-cultural approaches. In addition, such degree of autonomous behaviour may sometimes materialize in conflict leading to change in behaviour.

It should be noted that constructivism is not incompatible with socio-cultural approaches, although they are not strictly complementary (Packer and Goicoechea, 2000). In consequence, socio-cultural and constructivist aspects can be applied simultaneously to a given learning design.

\section{Different Ontological Notions of Change}

In the basic communication ontology described above, change can be considered a monotonous increase in the amount of information stored in each individual's mind. To put it in abstract, set-theoretic terms, we can say that change after a learning activity can be measured as the difference between the information stored at the previous moment $i-1$ and at the current moment $i$ - see expression (1).

$$
C_{0}(i)=\bigcup_{l \in \text { Learner }} I_{i}^{l}-I_{i-1}^{l}
$$

Information as stored in a learner's mind is considered as knowledge, so that change becomes an increase in individual knowledge. Learning designs are thus a plan of communication events, and their assessment can be stated in terms of the cardinality of the set $C_{0}(i)$. Consequently, the learning objects used for the communication in the case of e-learning are part of a communicative plan. This characterization evidences the main shortcomings of mere communication as a learning design. On the one hand, its scope is 


\section{Representation of change - ontologies of learning}

limited to considering the basic content of messages, and on the other hand, it precludes the consideration of the context of the learning activities beyond the analysis of noise. The obvious benefit is the ease of measuring change, although in a rather incomplete perspective. As a derivative of this simple characterization of change, the contribution of learning objects can be measured by counting the times they have serve as messages facilitating communication events to produce a learning outcome. Nonetheless, this simple notion is still valid for many training options, in which the learning experience is chiefly structured around the provision of some contents oriented to autonomous and individual learning.

The activity-centred ontology provides a much richer framework for learning, but its general-purpose nature results in the lack of a concrete definition for change. This is a result of the generic nature given to learning objectives. It allows for "machine-readable specifications” for them, and also explicitly considers competencies as objectives, but it is deliberately open to other kind of specifications. The use of competencies allow for a consideration of change as a (partial or not) covering of some form of “competency gap”, as described by Sicilia (2005). Such consideration of competencies shifts the emphasis on information storage in learner's mind to observable behaviour in work situations. In addition, relationships between competencies can be considered, which leads to a more elaborated version of expression (1). But the essential element describing change in this framework is that of Outcome. Nonetheless, the generic nature of LD entails that this notion of outcome as that specified by learning objectives is deliberately open. For example, the fact that "one student completes an activity" can be recorded as an outcome, which says little about the underlying assumptions of change. In fact, the specification of LD refers to the IMS Learner Information Packaging (LIP) specification as the model for recording outcomes. LIP can be used to record certifications, licenses and academic achievements and also competencies, loosely described as "skills, knowledge, and abilities acquired in the cognitive, affective, and/or psychomotor domains". In consequence, the range of changes that can be recorded is broad, but there is not an explicit consideration of any learning ontology.

Constructivism introduces a new dimension in the characterization of change, since the outcome of learning becomes a change in a knowledge structure, which is in principle much more difficult to assess than approaches centred on simple storage or in observable behaviour. In any cases, learning activities are in this framework considered as knowledge structure modification enablers, i.e. activities may trigger some kind of change in the knowledge structure of the learner, being such change additive or not, since re-structuring of knowledge is an important element under this view.

If a semantic-network representation like topic maps ${ }^{3}$ is assumed as the structure of human knowledge (which is far from being universally accepted), then change should be measured in terms of changes in the fundamental ontological commitments of such representation (Davis, Shrobe \& Szolovits, 1993). For example, expression (2) provides a simple model for such measurement based on topic $(T)$ and association $(A)$ changes (subscripts $N$ for “new” and $D$ for “deleted”).

$$
C_{A}(i)=T_{N} \cup A_{N} \quad C_{D}(i)=T_{D} \cup A_{D}
$$

But the problem of measuring change becomes even more complicated if axioms or other kinds of representational elements are taken into account. In addition, such simple model do not consider that some changes in structure are more influential than others, 


\section{M.A. Sicilia \& M. Lytras}

since they open new perspectives to practice or change significantly a whole parcel of one's representation of the world. This is why even for constructivist designs it is often considered appropriate to adopt a behavioural account of assessment, based, for example, on competencies. It should be noted that in expression (2) it is not assumed that change is monotonous, since some structure changes may entail breaking associations or discarding topics.

An alternative view described by Jonaasen (1991) states that it is the process of knowledge acquisition which should be evaluated, not any product or observable behaviour. This significantly shifts the focus on outcomes or knowledge representations to a detailed record of the activity of learners. Such record can be considered as raw data that requires the interpretation of tutors to come up with a figure of the actual change that took place. While this provides a rich framework, it dramatically increases the effort spent in evaluation.

Change is connected also to Identity (Packer, 1995) in socio-cultural approaches. This introduces a new dimension in the assessment of change inside organizations i.e. that of learning as a driver for change in positions inside the organization or group. Such view leads to explicitly representing changes in positions and/or responsibilities as the motivation for learning activities, making the functional structure of the organization an integral part of learning programs, and giving a prominent role to mentoring. In addition, the assessment of change in socio-cultural approaches relates mostly to the competency of individuals to interact and collaborate inside a group, so that social interactions should be recorded and be subject to inquiry. Approaches to social network analysis are useful tools for such assessments of change. Reffay and Chanier (2003) provide an illustration of such kind of analysis. In any case, a detailed assessment program driven by a sociocultural ontology would require measuring "historical change" inside groups, which becomes practically unfeasible in the case of learning programs in which the group is typically a temporary arrangement of individuals with no subsequent cooperation at work.

If we take the perspective of organizational change, it is evident that only the sociocultural ontology completely addresses the main elements of change in behaviour that is stressed in approaches to learning organizations (Reynolds and Ablett, 1998). This is due to the fact that such behaviour is characteristic of the emerging behaviour of groups inside the organizational environment, and not simply of an aggregate of individual learners isolated from each other. Culture plays a significant role in organizational change (Örtenblad, 2001; Lakomski, 2001), and in consequence, learning systems that are intended to support it should somewhat represent it inside their ontological structures. Nonetheless, this is a complex issue for which current learning specifications are not yet prepared. The activity-centred paradigm of LD, for example, is not intended to reflect changes in methods, but only to represent them statically and isolated from the others. Another notable lack in such specifications is that of structures to describe social relationships and roles that go beyond concrete role-playing in directed activities. The move to more complex ontological frameworks for interpreting change requires the integration of interpretive and subjective notions that go beyond a positivist view of learning activities, similar to those described by Solem (2003) for the field of logistics. 


\section{Representation of change - ontologies of learning}

\section{Conclusions and implications for further research}

The generic ontologies of learning described so far evidence the heterogeneity in notions of change that are derived from diverging theoretical positions. Although an activity-centred representation is flexible enough to accommodate the resulting design of diverging ontologies of learning, more specific elements are required to explicitly accommodate fundamental elements that are important from the viewpoint of specific assumptions about the nature of learning. Concretely, constructivism introduce an emphasis in the representation of knowledge structures and on a more independent and active role of the learner, and socio-cultural approaches focus on different roles of learners inside a group with specific characteristics and roles, and introduces the notion of identity and social relationship as important issues.

The accounts of change vary significantly with the approach used. Naive information processing assumptions lead to monotone models of storage, as simplifications of richer models based on observable behaviour in the form of competencies. Constructivism requires accounts of structural change if learner's knowledge is used as the object to measure, and even more elusive notions depending on the epistemology attributed to changes in knowledge. In addition, the socio-cultural view introduces change in identity and the assumption of responsibilities and acquisition of culture and relationships as elements of change in learning.

What becomes clear after the described analysis is that the notions and methods of assessment of change widely vary depending on the ontological assumptions held in the process of designing the learning program or activity. In consequence, there is a link between the rationale and the final assessment of learning designs, which constitutes a sound motivation to explicitly represent those assumptions in the form of ontological representations. The field of learning technology is not neutral to these different theoretical assumptions, since computer-based learning environments must eventually produce some form of recording of the outcomes of learning designs. Such outcomes are essentially the changes produce in learners and in their context, and the accounts of change vary significantly depending on the position. Recording only competencies is a solution that focuses on external observable behaviour, but it can not be used as a source of inquiry about the appropriateness of different theoretical positions on learning, since it ignores the details of the knowledge or social elements that changed and produced as a result the change in competency level.

Further research should elaborate the directions sketched here in several dimensions, including the following ones:

- Much work is required in elaborating ontologies of different theoretical positions of learning, beyond flexible activity-centred and general-purpose languages like IMS-LD.

- From a practical perspective, there is a need to provide compact machinereadable representations for the descriptions of the ontological assumptions taken into account in concrete learning designs.

- The notions of change attached to the different ontological positions require a clarification for the sake of assessment and measurement, reaching ideally the state of "accountable" metrics for intellectual capital.

This paper has provided a first analysis for the first of the just mentioned dimensions of research. The authors hope that this motivates further efforts in the near future. 


\section{M.A. Sicilia \& M. Lytras}

\section{References}

1. Allert, H. (2004). Coherent Social Systems for Learning: An Approach for Contextualized and Community-Centred Metadata. Journal of Interactive Media in Education, 2004 (2).

2. Appelbaum, S.H., St-Pierre, N. and Glavas, W. (1998). Strategic organizational change: the role of leadership, learning, motivation and productivity. Management Decision 36/5, 289-301

3. Baader, F., Calvanese, D., McGuinness, D., Nardi, D., Patel-Schneider, P. (eds.). (2003). The Description Logic Handbook. Theory, Implementation and Applications, Cambridge.

4. Barros, B., Verdejo, M.F., Read, T. and Mizoguchi, R. (2002). Applications of a Collaborative Learning Ontology. In Proceedings of MICAI'2002 Mexican International Conference on Artificial Inteligence, Springer-Verlag, pp. 301-310

5. Berners-Lee, T., Hendler, J., Lassila, O. (2001). The Semantic Web. Scientific American, 284(5), 34-43.

6. Christensen, W.D. and C.A. Hooker (2000). An interactivist-constructivist approach to intelligence: self-directed anticipative learning. Philosophical Psychology, vol. 13, no. 1, pp.545.

7. Davis, R., Shrobe, H. and Szolovits, P. (1993). What is a Knowledge Representation? AI Magazine, 14(1):17-33.

8. EML (2000). Educational Modelling Language. Retrieved October 14, 2003, from http://eml.ou.nl

9. García, E. \& Sicilia, M.A. (2003). User Interface Tactics in Ontology-Based Information Seeking . Psychnology e-journal 1(3):243-256.

10. Gruber T. (1995). Towards principles for the design of ontologies used for knowledge sharing. International Journal of Human-Computer studies, 43 (5/6), 907 - 928.

11. Holsapple, C.W. and Joshi, K.D. (2004). A formal knowledge management ontology: Conduct, activities, resources, and influences. Journal of the American Society for Information Science and Technolog\}, 55(7): 593--612.

12. Inaba, A., Supnithi, T., Ikeda, M., Mizoguchi, R. \& Toyoda, J. (2000). An Overview of "Learning Goal Ontology". In Proceedings of the Analysis and Modelling of Collaborative Learning Interactions Workshop at ECAI 2000.

13. Jonassen, D. (1991). Objectivism vs constructivism: Do we need a new philosophical paradigm? Educational Technology, Research and Development, 39(3), 5-13.

14. Jonassen, D.H. and Wang, S. (1993). Acquiring Structural Knowledge from Semantically Structured Hypertext. Journal of Computer-Based Instruction, 20(1), 1-8.

15. Koper, R. (2004). Use of the Semantic Web to Solve Some Basic Problems in Education: Increase Flexible, Distributed Lifelong Learning, Decrease Teacher's Workload. Journal of Interactive Media in Education, 2004 (6).

16. Lakomski, G. (2001). Organizational change, leadership and learning: culture as cognitive process. The International Journal of Educational Management, 15/2, 68-77.

17. Lave, J. and E. Wenger (1990). Situated Learning: Legitimate Peripheral Participation. Cambridge, Cambridge University Press.

18. LD (2003). IMS Learning Design. Information Model, Best Practice and Implementation Guide, Binding document, Schemas. Retrieved October 3, 2003, from http://www.imsglobal.org/

19. Noy, N. and Musen, M. (2003). The PROMPT suite: interactive tools for ontology merging and mapping. International Journal of Human-Computer Studies, 59(6), 983-1024.

20. Ordóñez de Pablos, P. (2004). A guideline for building an intellectual capital statement: the 3R model. International Journal of Learning and Intellectual Capital, 1(1), 3-18.

21. Örtenblad, A. (2001). On differences between organizational learning and learning organization. The Learning Organization, 8(3), pp. 125-133. 


\section{Representation of change - ontologies of learning}

22. Packer, M. and Goicoechea, J. (2000). Sociocultural and constructivist theories of learning: ontology, not just epistemology. Educational Psychologist, 35(4), 227-241.

23. Packer, M. and Hwang, J. (1999). School and ontological change. Proceedings of the Annual Meeting of the American Anthropological Association, Chicago.

24. Packer, M. (1995). "Local ontology" - cultural work in the classroom. In Proceedings of the AERA annual meeting, San Francisco.

25. Polsani, P. R. (2003). Use and Abuse of Reusable Learning Objects. Journal of Digital information, 3(4).

26. Reffay, C., Chanier, T. (2003). How social network analysis can help to measure cohesion in collaborative distance-learning. "Computer Supported Collaborative Learning". Bergen: Kluwer Academic Publishers.

27. Reynolds, R. and Ablett, A. (1998). Transforming the rhetoric of organizational learning to the reality of the learning organization. The Learning Organization, 5(1), pp. 24-35.

28. Sicilia, M.A. (2005). Ontology-Based Competency Management: Infrastructures for the Knowledge-intensive Learning Organization. In: Lytras and Naeve (Eds.): Intelligent Learning Infrastructures in Knowledge Intensive Organizations: A Semantic Web perspective. IDEA, USA (to appear, 2005).

29. Sicilia, M.A., García, E., On the Convergence of Formal Ontologies and Standardized eLearning. Journal of Distance Education Technologies 2(4) (to appear, Oct 2004)

30. Solem, O. (2003). Epistemology and Logistics: A critical overview. Systemic Practice and Action Research, 16, pp. 437-454

31. Stutt, A. and Motta, E. (2004). Semantic Learning Webs. Journal of Interactive Media in Education, 2004 (10).

32. Weaver, W. and Shannon, C.E. (1949). The Mathematical Theory of Communication, Urbana, Illinois: University of Illinois Press.

\section{Notes}

1. Here we use the term "learning system" instead of the common term "learning management system" to refer to systems that include learning technology but also structures and processes that are social or human in nature.

2. http://protege.stanford.edu/

3. http://www.topicmaps.org/ 